\title{
COMPORTAMENTO DO CONSUMIDOR DE TECNOLOGIA VESTÍVEL: CARACTERÍSTICAS QUE INFLUENCIAM NA INTENÇÃO DE CONSUMO ${ }^{1}$
}

\author{
Lorena Renata Costa Cantanhede ${ }^{2}$ \\ Edgar José Pereira Dias ${ }^{3}$ \\ Igor de Jesus Lobato Pompeu Gammarano ${ }^{4}$ \\ Emílio José Montero Arruda Filho ${ }^{5}$
}

http://dx.doi.org/10.1590/1413-2311.225.85428

\section{RESUMO}

O presente estudo trata sobre as características que influenciam a intenção de consumo de produtos tecnológicos vestíveis, baseado nos valores hedônicos, utilitários e de status social. O objetivo é compreender como se dá o processo de consumo dos relógios inteligentes (Apple Watch), identificando as características de compras dos usuários, dado a pouca prática tecnológica e percepção da utilidade do produto. Realizou-se uma pesquisa de campo, com a finalidade de analisar a intenção de consumo dos usuários, com aplicações de questionários abertos com imagem e características das funcionalidades do produto, nos quais os usuários podiam expressar abertamente suas opiniões para que em seguida fosse realizado a análise do conteúdo dos comentários. Os resultados obtidos apontam a existência de uma ligação entre fatores comportamentais como hedonismo, utilitarismo, status social, risco social, inveja e cobiça e sua influência quanto ao uso e consumo do relógio inteligente Apple Watch.

\footnotetext{
${ }^{1}$ Submetido em 09/08/2018, aprovado em 06/11/2018.

2 Universidade da Amazônia (UNAMA) - Bacharelado em Administração; Belém - PA (Brasil) lorena.renata@hotmail.com

${ }^{3}$ Universidade da Amazônia (UNAMA) - Programa de Pós-graduação em Administração - PPAD; Belém - PA (Brasil) - edgarjose.dias@gmail.com

${ }^{4}$ Universidade da Amazônia (UNAMA) - Programa de Pós-graduação em Administração - PPAD; Belém - PA (Brasil) - igor.internacionalista@gmail.com

5 Universidade da Amazônia (UNAMA) - Programa de Pós-graduação em Administração - PPAD / Universidade FUMEC - Programa de Doutorado e Mestrado; Belém -PA (Brasil) - emilio.arruda@hotmail.com
} 
Palavras-Chave: Hedonismo. Valor utilitário. Valor social. Intenção de consumo.

\title{
CONSUMER BEHAVIOR OF WEARABLE TECHNOLOGY: CHARACTERISTICS THAT INFLUENCE CONSUMPTION INTENTION
}

\begin{abstract}
This paper presents a study about the characteristics that influence the consumption intention of wearable technological products, based on hedonic, utilitarian and social status values. The objective is to understand how the consumption process of intelligent watches (Apple Watch) occurs, identifying the shopping characteristics of users, given the little technological practice and perception of the usefulness of the product. For the development of the research, a field research was conducted to analyze the users' intention to consume, with the use of questionnaires with open questions that included image and features of the product functionalities, in which users could openly express their opinions. Content analysis whas then performed. The results indicated the existence of link sbetween behavioral factors such as hedonism, utilitarianism, social status, social risk, envy and greed and their influence on the use and consumption of the intelligent Apple Watch.
\end{abstract}

Keywords: Hedonism. Utilitarian value. Social value. Intention of consumption.

\section{COMPORTAMIENTO DEL CONSUMIDOR DE TECNOLOGÍA VISIBLE: CARACTERÍSTICAS QUE INFLUYEN EN LA INTENCIÓN DEL CONSUMIDOR}

\section{RESUMEN}

Este trabajo presenta un estudio de las características que influyen en la intención de consumo de productos de tecnología visible, basado en el valor hedónico, servicios públicos y el estatus social. El objetivo es entender cómo es el proceso de consumo de relojes inteligentes (Apple Watch), la identificación de las características comerciales de los usuarios, dado poca práctica tecnológica y la percepción de la utilidad del producto. Para el desarrollo de la investigación presentada era una investigación de campo, con el fin de analizar la intención de consumo de los usuarios, con aplicaciones de cuestionarios abiertos con la imagen y las características de las características del producto, donde los usuarios 
pueden expresar abiertamente sus puntos de vista. En la continuación, se llevó a cabo el análisis del contenido. Los resultados obtenidos indican la existencia de una ligación entre factores comportamentales como hedonismo, utilitarismo, status social, riesgo social, envidia y codicia y su influencia en el uso y consumo del reloj inteligente Apple Watch.

Palabras clave: El hedonismo. El valor de uso. Valor social. Uso previsto.

\section{INTRODUÇÃO}

O mercado de tecnologias se desenvolveu de maneira ágil, o que concedeu ao consumidor diversas opções de equipamentos e dispositivos tecnológicos que pudessem satisfazer suas necessidades e seus desejos (DHAR, 2018; TENUTA; TESTA, 2018). Neste cenário, surgiram equipamentos tecnológicos cotidianamente presentes na vida do ser humano, de forma compacta e convergente (GILL, 2008; HARRIS; BLAIR, 2006; KIM; WONJOON, 2018; LEE; PARK; KANG, 2018), possibilitando novas formas de uso e estimulando a atualização dos consumidores para os produtos com diversos características e serviços disponíveis.

Nesta nova configuração tecnológica, surgem os produtos convergentes, fazendo junções como serviço de televisão, jogos, redes sociais, acesso à internet, dentre outras opções tecnológicas disponíveis nos aparelhos celulares (GILL, 2008; HARRIS; BLAIR, 2006), os quais quando unificados tornam-se um só dispositivo com multifuncionalidade.

Além disso, inovações foram exigidas por necessidades específicas dos consumidores, que passam a ser mais rigorosos. Logo, foi demandada uma resposta mais inovadora do setor tecnológico adaptada ao cotidiano intenso de indivíduos, que não buscam somente utilizar os dispositivos, mas também se diferenciar socialmente por intermédio da marca, das funcionalidades e do design destes produtos (NIERODA; MRAD; SOLOMON, 2018).

Baseado nestas necessidades surgem às tecnologias vestíveis (Wearable Technologies) (DEHGHANI; DANGELICO, 2018; NASCIMENTO; OLIVEIRA; TAM, 2018; GUPTA; RODRIGUES; MATHEW, 2018), sendo estas definidas como dispositivos utilizados como acessórios estéticos que podem ser combinados com peças de roupas. Estes dispositivos também oferecem funções como transmissões de dados, acesso à Web, acompanhamento de funções vitais do corpo humano, dentre outras funcionalidades convergidas em um único equipamento (SCHERER, 2014). 
As tecnologias vestíveis são concebidas na forma de pulseiras, relógios, óculos, camisas, sapatos, dentre outros (DEHGHANI; DANGELICO, 2018; NASCIMENTO; OLIVEIRA; TAM, 2018; GUPTA; RODRIGUES; MATHEW, 2018). Deste modo, pode ser observado como exemplo o dispositivo denominado Google Glass da empresa Google que teve seu produto descontinuado antes mesmo de entrar no mercado (NUNES; ARRUDA FILHO; 2018). Tal fato se deu devido ao risco percebido pela sociedade em relação a violação da privacidade e ao não cumprimento da lei de direitos autorais (HEIN et al., 2018).

Neste cenário, o foco deste estudo é referente ao produto Apple Watch, um dispositivo tecnológico no formato de relógio de pulso, que agrega diversas funcionalidades, como acesso à internet, realização de ligações, leitura de e-mails, ou seja, é um dispositivo multifuncional que se destaca como vestimenta (PAGE, 2015).

Esta convergência presente nos produtos tecnológicos apresentados possui a diversificação dos atributos que geram diferentes conceitos e valores, sendo os responsáveis pela motivação e excitação de consumo, como as características utilitárias, hedônicas e sociais, presentes na convergência. O valor utilitário é definido em função das características de racionalidade e instrumentalidade do produto, servindo para aplicações fundamentais ao desenvolvimento pessoal, da empresa ou sociedade (OKADA, 2005; SLAMA; SINGLEY, 1996). Quando as características propõem relações emocionais com atributos voltados ao prazer, diversão e satisfação define-se este contexto como hedônico. O valor social diferente do hedônico traz o modismo, a individualidade e o compartilhamento do sucesso, posse e usos que aquele indivíduo alcançou ou faz parte (KATZ; SUGIYAMA, 2006).

Sendo assim, o estudo busca responder a seguinte questão: Quais fatores influenciam na adoção de produtos tecnológicos do tipo vestíveis? Deste modo, um dos pontos fundamentais a serem discutidos nesta pesquisa é a relação de valores de compras hedônicas (prazer, satisfação e diversão), sociais (status, diferenciação e posicionamento), e utilitárias (qualidade, finalidade, etc.) por parte dos consumidores de produtos vestíveis, verificando de que forma os fatores de decisão motivam e instigam estes tipos de consumidores.

Logo, a escolha do Apple Watch levou em conta as características inovadoras e convergentes que atraem grupos específicos de usuários de tecnologia, os quais focam na utilização destes dispositivos em práticas esportivas, cuidados com a saúde e em elementos estéticos ligados a moda e a cultura fashion (WOOD, 2017), a fim de combinar com a vestimenta que tende a influenciar os usuários do ponto de vista social (NIERODA; MRAD; SOLOMON, 2018; WOOD, 2018). 
A coleta de dados do estudo foi realizada por meio de um modelo de questionário aberto (AMADO, 2013), no qual os entrevistados pudessem expor livremente suas opiniões sobre questões mais amplas do conceito de uso do produto. A análise dos dados coletados se deu por intermédio da análise de conteúdo (BARDIN, 1977), que buscou compreender os significados dos comportamentos e preferências dos indivíduos consumidores de tecnologias vestíveis de forma profunda.

Sendo assim identificado neste contexto como fator hedônico, ações executadas com a finalidade de se obter prazer e diversão como resultado (ARRUDA FILHO; DHOLAKIA, 2013). Já os fatores utilitários estão direcionados às funções que facilitem atividades laborais e a execução de tarefas específicas por seus usuários, no qual o consumidor possui decisões racionais para sua escolha (FARIAS; DUSCHITZ; CARVALHO, 2014).

O fator social pode ser definido como a satisfação pelo status e conservação do ego e da imagem, visto que os usuários de tecnologia utilizam seus dispositivos como uma forma de expressão simbólica, que traduz a sua identidade social com o objetivo de se diferenciar em um determinado contexto coletivo (ARRUDA FILHO, CABUSAS E DHOLAKIA, 2008; NIERODA; MRAD; SOLOMON, 2018).

Este estudo divide-se da seguinte forma: primeiro é fundamentado a partir de conceitos e teorias relacionados a produtos convergentes com enfoque no hedonismo, utilitarismo e valor social. Em seguida, realiza-se a discussão sobre o tema proposto levando em consideração as intenções de consumo dos usuários do Apple Watch. No tópico seguinte, é descrita a sistemática ligada a metodologia, destacando a ocorrência da coleta e análise dos dados utilizados para a avaliação do fenômeno estudado, visto que os resultados são discutidos em forma de categorias de usuários para as tecnologias vestíveis (DEHGHANI; DANGELICO, 2018). Por fim, o trabalho gera conclusões ligadas aos pontos interpretados, também sendo indicadas as limitações do estudo e propostas de trabalhos futuros que podem vir a ser desenvolvidos a partir dos resultados alcançados.

\section{COMPORTAMENTO DE CONSUMO E TECNOLOGIAS VESTÍVEIS}

As inovações tecnológicas passaram a ser substanciais na vida do ser humano, visto que o grau de utilidade destes dispositivos se tornou indispensável, pois diversos indivíduos utilizam dispositivos tecnológicos como uma extensão de seus Selfs (BELK, 1988), inserindo-os como parte imprescindível de suas rotinas (LORENZETTI et al., 2012), gerando em alguns casos, dependência em relação ao seu uso (MICK; FOURNIER, 1998). Para Hanneman et al. (1969) a difusão da inovação trata do processo a qual ela é comunicada REAd | Porto Alegre - Vol. 24 - No 3 - Setembro / Dezembro 2018 - p. 244-268 
diante de determinados canais de comunicação ao longo de um caminho para os membros de uma sociedade. Rogers (2003) descreve em seu modelo de difusão da inovação, como um sistema específico pode ser aplicado em sistemas semelhantes com a realidade social. Sendo assim, produtos ou serviços inovadores passam a ser mais convenientes quando no momento da adoção da inovação, o consumidor percebe e compreende as vantagens e compatibilidades com suas necessidades (JOEP et al., 2011).

Conforme Dias (2012), o processo de difusão da inovação passou a ter impulso pela capacidade de incorporação e agregação de múltiplas funções, sejam elas, por ferramentas ou serviços em um único produto (HARRIS; BLAIR, 2006). Sendo assim, a convergência tecnológica (HARRIS; BLAIR, 2006; GILL, 2008; KIM; WONJOON, 2018; LEE; PARK; KANG, 2018; NUNES et al., 2000) passou a ter um papel fundamental no processo de escolha e decisão para o consumidor.

Conforme Yoffie (1997), a convergência se trata de um novo paradigma contemporâneo de produtos eletrônicos com alta tecnologia, no qual se almeja agregar diversas funções em um mesmo dispositivo. Além disso, os consumidores também percebem os produtos convergentes como sendo menos arriscados, pois estes oferecem uma diversidade de opções tecnológicas aos seus usuários, em detrimentos dos dispositivos dedicados que apresentam apenas uma única função especifica (HARRIS; BLAIR, 2006).

Por meio da convergência tecnológica as empresas estão se questionando sobre quais características presentes dos aparelhos os consumidores possuem maior interesse em função de suas demandas, hábitos e experiências de consumo (GILL, 2008; HARRIS; BLAIR, 2006; NUNES et al., 2000). Nesse contexto preditivo dos atuais interesses dos consumidores, é estratégico para as empresas obterem vantagens no mercado competitivo, a partir de observações e identificações de tendências e impactos que novos produtos podem gerar nos consumidores, descrevendo assim quais perfis ou grupos possuem os mesmos valores de consumo (ARRUDA; MIRANDA, 2003).

Os produtos tecnológicos foram se modificando e oferecendo novos serviços e acessos de comunicação (NUNES; WILSON; KAMBIL, 2000). Os equipamentos do tipo, smartphones (telefones inteligentes), apresentam profundas integrações hedônicas que propõe diversidades satisfatórias aos consumidores, como: câmera integrada, acesso à internet, reprodução de músicas, acesso a e-mails, redes sociais, agenda, calculadora, dentre outras opções de serviços online (LEE; CHO, 2015).

A convergência tecnológica possui um grande atrativo para os consumidores, visto que a grande quantidade de opções integradas os motiva a consumirem, dado o pensamento 
de que quanto maior forem a quantidade de produtos e serviços integrados, mais estes indivíduos utilizarão o produto (NUNES et al., 2000). Ademais, os usuários também percebem que quanto mais atualizado, integrado e inovador for o produto, mais vantajoso será consumir e possuir este equipamento (ARRUDA FILHO; CABUSAS; DHOLAKIA, 2008).

Quanto à intenção de compra dos consumidores de produtos convergentes destacamse dois fatores: o da compra motivada por questões hedônicas (prazer, diversão e satisfação) e a compra motivada por questões utilitárias (finalidades laborais e fatores racionais) (FARIAS; DUSCHITZ; CARVALHO, 2014; LEE; LEE; GARRET, 2013), na qual a compra por utilitarismo se caracteriza pela forma que será utilizado o produto, com critérios para o uso no trabalho, estudo ou atividades de necessidades básicas do indivíduo. Já o hedonismo possui características de uso ligadas ao prazer, diversão, entretenimento e satisfação (ARRUDA FILHO; CABUSAS; DHOLAKIA, 2008; ARRUDA FILHO; DHOLAKIA, 2013).

Okada (2005) indica que os consumidores adquirem confiança para sua compra quando os produtos possuem características utilitárias, pois as compras motivadas por questões hedônicas necessitam de justificativas para amenizar a culpa do consumo. Sendo assim, quando se efetua uma compra utilitária não existe a necessidade de se justificar em função da finalidade do uso com o produto.

Arruda Filho, Cabusas e Dholakia (2008), indicam que a utilização de um dispositivo tecnológico com características hedônicas possui também atributos do tipo utilitário social, no qual este fator está relacionado a um produto que sua funcionalidade seja gerar o status ou indicar aos demais indivíduos sobre o posicionamento social do seu usuário.

Segundo Okada (2005), quando falamos de produtos tecnológicos, o hedonismo indica emoção no consumo e no processo de posse, sendo assim, o consumo pode apresentar uma tendência hedônica e ao mesmo tempo social como forma de desejo, sem a relação com as características utilitárias, visto que o consumidor se utiliza destas características de posicionamento como justificação para o uso. Os consumidores estão utilizando produtos como objetos pessoais comumente utilizados, como partes estendidas de seus corpos e roupas (KATZ; SUGIYAMA, 2006).

Já para Ehrenberg (2012), os valores ligados ao hedonismo, utilitarismo e ao status social são executados pelo homem há muitos séculos, tanto para suprir suas necessidades, como também para se destacar na sociedade. Na sociedade contemporânea, o consumo de inovações tecnológicas cresce, fazendo com que o consumo comece a ser direcionado para o 
contexto do valor social (KATZ; SUGIYAMA, 2006), uma diferenciação na sociedade para despertar interesses dos seus produtores devido aos diversos atributos hedônicos, utilitários e sociais intrínsecos a estes equipamentos.

Contudo, os indivíduos tornaram-se mais exigentes e buscam vantagens, o que gerou a intensificação do consumo de produtos convergentes em detrimento de equipamentos dedicados que possuem apenas uma função específica (EHRENBERG, 2012; GILL, 2008; HARRIS; BLAIR, 2006; KIM; WONJOON, 2018; LEE; PARK; KANG, 2018; NUNES et al., 2000 ).

A convergência é mensurada pelos atributos que são incluídos no produto, podendo estes serem hedônicos ou utilitários, e até mesmo sociais (LEE; LEE; GARRETT, 2013). O consumo utilitário é descrito desde a teoria da ação racional de Ajzen e Fishbein (1980) e por Holbrook e Hirschman (1982) em seus estudos sobre estes conceitos emocionais e racionais no consumo, onde se pressupõe que o consumidor em meio a processos alternativos de consumo busca pela melhoria e efetividade dos serviços ficando em sua funcionalidade e racionalidade.

Entretanto, Okada (2005) relaciona o quanto vivenciar as situações prazerosas, leva a uma preferência e predisposição / atitude ao contexto hedônico, que, entretanto, possui em meio ao cenário social de convivência, situações de culpa. Quando consumidores são expostos ao consumo hedônico, ele é facilmente aceito e consumado, entretanto em um contexto de escolha com duas opções de consumo sendo uma hedônica e outra utilitária, por mais que a preferência seja pela hedônica, caso não exista justificação racional ao consumo, a escolha final será pela utilitária, dado o sentimento de culpa envolvido com o hedonismo e altos custos sem razão apropriada (OZCAN; SHEININ, 2015).

Segundo Arruda Filho, Cabusas e Dholokia (2008), possuir uma tecnologia avançada, com multifuncionalidade, faz com que os consumidores fiquem satisfeitos tanto por existir a utilidade do produto, quanto pelo hedonismo apresentado neste, pois este consumo possibilita um bom posicionamento na mente dos consumidores tendo a união de utilidade e de prazer. Katz e Sugyiama (2006) indicam que a moda é um dos motivos de compra do consumidor, pois o consumo de tecnologias que aprofundam aspectos ligados ao modismo, proporciona status e prestígio na sociedade.

Logo, Ehrenberg (2012) comenta que as marcas são responsáveis por proporcionar aos consumidores elementos que possam lhes diferenciar socialmente. Por exemplo, o consumidor que adota um produto da marca Apple, além da possibilidade de usufruir da convergência de diversos produtos e serviços integrados nos dispositivos (GILL, 2008; 
HARRIS; BLAIR, 2006; NUNES et al., 2000;), também podem dispor de características ligadas ao design, conceito e reputação da marca, funcionalidade e diferenciação social por meio do valor da marca e do elevado preço de mercado dos produtos.

Com as tendências da moda, as inovações estão sendo combinadas com peças de vestuário, adaptando-as por meio da inclusão de elementos tecnológicos e informacionais, o que ocasiona mudanças relacionadas a forma como os usuários destas tecnologias expressam aspectos ligados as suas personalidades (BELK, 1988), além de modificar a forma como estes indivíduos se comunicam interpessoalmente (PANISSON, 2009), seja no ambiente online (BELK, 2013) ou off-line (BELK, 1988) por intermédio da extensão de seus Selfs no ambiente digital (BELK, 2013).

Deste modo, é identificado na literatura que a inclusão de tecnologias, como uma peça no vestuário, desperta o interesse dos indivíduos baseado em aspectos emocionais, conectados ao uso de dispositivos portáteis, visto que sua utilização influencia suas percepções ligadas à interação social e ao uso funcional, concedendo diversas sensações visuais e sonoras (PANISSON, 2009).

Devido ao novo contexto tecnológico convergente, houve a possibilidade de ascensão das "tecnologias vestíveis". Assim, tais dispositivos foram desenvolvidos para serem adaptados ao cotidiano de seus usuários, levando em consideração suas rotinas de trabalho e lazer. Tal fato facilita a execução de tarefas cotidianas e gera status social por intermédio da integração entre designs, que se destacam pelos aspectos estéticos ligados a moda e a inovação tecnológica (BARRADAS et al., 2015).

Segundo Donati (2004), o que difere a tecnologia vestível de uma tecnologia comum é o fato da primeira possuir características ligadas à utilização integrada ao vestuário do indivíduo (NASCIMENTO; OLIVEIRA; TAM, 2018), como uma peça de roupa que se fixa junto ao corpo possibilitando o acesso de informações vitais do usuário e do ambiente no qual este está inserido. A ideia é que esses produtos possam fazer parte do cotidiano das pessoas comuns, despertando o desejo de se obter o produto com uma interação corrente (FRANCO, 2013).

As características de design e as funções disponíveis no dispositivo Apple Watch, gradativamente tem instigado o desejo dos consumidores, visto que as opiniões dos consumidores têm se modificado, de argumentos declarados como "é bom ter este equipamento" para "é algo que preciso ter" (ARAUJO, 2015).

Então a análise do interesse dos usuários por tecnologias vestíveis, está relacionada com as características hedônicas, utilitárias e de valor social. Baseado nisto, a análise destes 
aspectos se mostra relevante, com foco no dispositivo Apple Watch para que se compreenda quais os elementos e valores estão envolvidos no processo de escolha e avaliação desta categoria de produtos, levando em consideração valores hedônicos (relação emocional), utilitários (relação racional) e sociais (relação entre o emocional e racional) no consumo, tendo a presença de diferenciações de inovações tecnológicas vestíveis em função das integrações e convergências tecnológicas (DONATI, 2004).

\section{METODOLOGIA}

A presente pesquisa buscou compreender os fatores que influenciam na adoção de tecnologias vestíveis (DEHGHANI; DANGELICO, 2018; GUPTA; RODRIGUES; MATHEW, 2018; NASCIMENTO; OLIVEIRA; TAM, 2018), em especial, quanto ao uso do dispositivo Apple Watch, a partir de um estudo qualitativo de caráter exploratório (FARIAS FILHO; ARRUDA FILHO, 2013).

Os principais termos pesquisados para atender essa pesquisa foram: convergência tecnológica, tecnologia vestível, comportamento do consumidor focado no hedonismo, utilitarismo e status social, tendo como objeto de pesquisa o dispositivo Apple Watch. O presente estudo foi construído tendo como base o comportamento do indivíduo que abrange diversas áreas, subáreas e temáticas atuais, até mesmo o campo de estudo como os da psicologia, inovações tecnológicas e a área que análise a satisfação dos consumidores neste mercado constituído por um ambiente convergente.

A pesquisa é caracterizada como qualitativa devido à coleta de dados ser baseada em contextos, conteúdos e descrição das análises textuais analisadas (BOGDAN; BIKLEN, 1994). Logo, foi desenvolvido um questionário estruturado com questões abertas, tendo como objetivo incentivar respostas reflexivas e profundas (AMADO, 2013). Conforme a Tabela 1, tal questionário foi composto por dez questões discursivas que abordam conceitos baseados na literatura e em teorias discutidas neste estudo, como o hedonismo, utilitarismo e status social, dividindo-se em quatro perguntas relacionadas ao status social (dado a relação com o utilitarismo e hedonismo), duas perguntas envolvendo o utilitarismo, duas perguntas referente à percepção de uso, uma pergunta abordando os valores hedônicos (envolvendo as características gerais de prazer e satisfação) e uma pergunta relacionada à decisão de compra. Por fim, foram realizadas perguntas ligadas aos dados socioeconômicos dos participantes, tais como nome, idade, profissão, sexo, estado civil para assim se definir o perfil dos respondentes. 
A distribuição destes questionários se deu de maneira presencial por meio de material impresso, com o objetivo de abranger um maior número de pessoas possíveis. Os respondentes se caracterizaram por serem indivíduos com idades entre 18 e 40 anos, sendo estudantes universitários e profissionais residentes no município de Belém/ Pará, que comumente utilizam tecnologias móveis no seu dia a dia como smartphones, tablets, notebooks entre outros. O questionário continha uma imagem do dispositivo para que os indivíduos entrevistados pudessem avaliar o produto em relação a sua utilidade e a predição de uso e consumo, a fim de obter informações sobre suas percepções relacionadas ao Apple Watch. Os questionários foram aplicados por um dos pesquisadores que realizou um processo de descrição detalhada do questionário, para depois deixar os participantes livres para explicarem suas percepções quanto ao produto inserido, dado o estímulo na apresentação inicial do instrumento de coleta. O Quadro 1 representa as informações ligadas aos dados primários extraídos durante a pesquisa realizada.

\section{Quadro 1 - Dados Primários da pesquisa do dispositivo Apple Watch}

\begin{tabular}{|c|l|}
\hline Descrição & \multicolumn{1}{c|}{ Informações da pesquisa } \\
\hline Total de questionários aplicados & 52 questionários abertos. \\
\hline Data da coleta desenvolvida & De 3 a 20 de novembro de 2015. \\
\hline Total de questões aplicadas & 10 questões sobre o dispositivo Apple Watch. \\
\hline Palavras-Chave & Hedonismo, Utilitarismo, Valor Social. \\
\hline
\end{tabular}

Fonte: elaborado pelos autores.

Foram coletados 52 questionários devidamente respondidos, visto que os questionários respondidos manualmente, foram transcritos com o objetivo de aperfeiçoar e facilitar a codificação do material coletado. Logo, os dados foram organizados no editor de texto Microsoft Word. Os dados foram inseridos com o objetivo de ocupar 2/3 de cada página do banco de dados, e o espaço restante referente à $1 / 3$, foi reservado para a codificação e análise manual dos materiais coletados. Colocaram-se as respostas por ordem lógica de cada indivíduo participante, sendo que ao término de cada resposta destacou-se a linha apontando o término de uma discussão e o início da próxima (questão avaliada). A Tabela 1 apresenta as bases teóricas que foram utilizadas para a construção das perguntas dos questionários aplicados durante o estudo.

O método de codificação utilizado neste trabalho foi baseado na análise de conteúdo (BARDIN, 1977), sem a utilização de software para tratar os dados coletados. Durante a execução do método, foram lidas as respostas dos participantes e destacado as palavras, frases ou trechos que expressassem os significados relacionados aos sentimentos, emoções e valores envolvidos no uso e no consumo do objeto de pesquisa estudado.

REAd | Porto Alegre - Vol. 24 - No 3 - Setembro / Dezembro 2018 - p. 244-268 
Tabela 1 - Perguntas presentes no questionário estruturado sobre o Apple Watch

\begin{tabular}{|c|c|c|c|}
\hline ORDEM & $\begin{array}{c}\text { BASE } \\
\text { TEÓRICA }\end{array}$ & PERGUNTA REALIZADA & $\begin{array}{l}\text { QUANTIDADE } \\
\text { DE } \\
\text { PALAVRAS } \\
\end{array}$ \\
\hline 1 & Hedonismo & $\begin{array}{l}\text { Explique quais as possibilidades de diversão e prazer que } \\
\text { você percebe em se utilizar o Apple Watch? }\end{array}$ & 889 \\
\hline 2 & Utilitarismo & $\begin{array}{c}\text { Descreva como você acredita que esse produto seria } \\
\text { necessário para você no seu dia-dia? }\end{array}$ & 964 \\
\hline 3 & Utilitarismo & $\begin{array}{l}\text { Explique como esse produto seria útil para o seu trabalho ou } \\
\qquad \text { seu estudo? }\end{array}$ & 862 \\
\hline 4 & Status Social & Explique como este produto traria valor pra você? & 666 \\
\hline 5 & Status Social & $\begin{array}{c}\text { Descreva quais sensações o uso deste produto entre seus } \\
\text { amigos e familiares lhe proporcionaria? }\end{array}$ & 541 \\
\hline 6 & Status Social & $\begin{array}{c}\text { Descreva como seria a reação dos seus amigos e familiares ao } \\
\text { lhe verem utilizando um produto deste tipo? }\end{array}$ & 664 \\
\hline 7 & Status Social & $\begin{array}{l}\text { Explique como este produto lhe traria diferenciação entre seus } \\
\text { amigos e familiares? }\end{array}$ & 746 \\
\hline 8 & Percepção de uso & $\begin{array}{l}\text { Explique o que poderia levar você a tomar uma decisão de } \\
\text { compra de um produto como este? }\end{array}$ & 632 \\
\hline 9 & Percepção de uso & $\begin{array}{l}\text { Descreva qual a percepção que você tem em ver uma pessoa } \\
\text { fazendo uso do Apple Watch? }\end{array}$ & 638 \\
\hline 10 & $\begin{array}{l}\text { Decisão de } \\
\text { compra }\end{array}$ & $\begin{array}{l}\text { Você compraria o Apple Watch pela marca e/ou por ser um } \\
\text { dispositivo inovador com multifuncionalidades ou por outros } \\
\text { motivos? Explique quais motivos. }\end{array}$ & 813 \\
\hline Total & & & 7415 \\
\hline
\end{tabular}

Fonte: elaborado pelos autores.

Após o término da primeira etapa de análise, que consistiu na seleção das palavras, frases ou trechos que possuíram maior relevância sobre o assunto, iniciou-se uma segunda fase na qual se codifica e depois se categoriza os usuários, conforme as teorias de base e as percepções de uso do produto pelos participantes das entrevistas.

Deste modo, os grupos oriundos das interpretações, foram destacados mediante ao contexto no qual se propuseram a defender, participar, se posicionar ou mesmo justificar, e suas percepções relacionadas a importância para seu uso e aceitação são descritas na análise dos resultados.

\section{ANÁLISE DOS DADOS}

Com base nos elementos coletados dos entrevistados, foram considerados e avaliados os fatores que melhor descreveram as influencias no comportamento do consumidor de tecnologia vestível (DEHGHANI; DANGELICO, 2018; NASCIMENTO; OLIVEIRA; TAM, 2018; GUPTA; RODRIGUES; MATHEW, 2018). Para se observar os fatores que influenciaram no comportamento de consumo, foi necessário dividir em diversas categorias 
de acordo com a que cada grupo defende, participa ou se posiciona conforme sua percepção de usuário tecnológico.

Tais categorias buscam descrever os comportamentos e preferências destes consumidores, relacionando suas observações com as teorias trabalhadas neste estudo, ou seja, o perfil de cada usuário em função dos conceitos que explicam as características de interesse. Além disso, a análise também conta com a transcrição dos trechos que refletem os comportamentos observados, sendo estes codificados indicando pontualmente a pergunta em que a resposta foi gerada, seguido pelo número correspondente ao participante da pesquisa.

\subsection{A PERCEPÇÃO HEDÔNICA E A JUSTIFICAÇÃO UTILITÁRIA RELACIONADA} AO USO DO APPLE WATCH.

Quando falamos de diversão e prazer ao se consumir um determinado produto, estamos identificando o hedonismo, ainda que os usuários justifiquem sua compra hedônica por fatores utilitários para assim diminuir sua culpa (HOLBROOK; HIRSCHMAN, 1982; OKADA, 2005). Em uma escala unidimensional, o hedonismo e o utilitarismo não são tratados de forma separada (VOSS et al., 2003).

Sendo assim, as atribuições de funcionalidades e prazer passam a gerar um significativo papel na adoção e no uso de novas tecnologias (CHITTURI et al., 2007). Diante disso, o processo de aceitação tecnológica depende das características hedônicas, sem excluir a possibilidade de uso das características utilitárias convergidas no dispositivo, pois o utilitarismo passa a ter um papel fundamental na justificação de consumo por parte do consumidor (ARRUDA FILHO; DHOLAKIA, 2013).

Utilizar um equipamento para a prática de esporte, como corridas e caminhadas através do GPS, sem se esquecer dos cuidados com a saúde pelo monitoramento cardíaco. (Pergunta 1; respondente 7)

Substitui o celular trazendo a possibilidade de ser usado também como acessório de beleza. (Pergunta 1; respondente 25)

A interação entre o homem e a tecnologia já é divertido, o que proporciona descontração em momentos de plena atividade física elou no dinamismo do trabalho. (Pergunta 1; respondente 50)

Possibilitaria o uso de games e outros itens da web. (Pergunta 1; respondente 15)

REAd | Porto Alegre - Vol. 24 - No 3 - Setembro / Dezembro 2018 - p. 244-268 
Os usuários apresentam diversas percepções sobre o dispositivo Apple Watch, destacando funções como jogos e reprodução de músicas, com o intuito de buscar prazer e influenciando assim na sua percepção ligada a compra do dispositivo. Porém outros justificam seu consumo hedônico pela característica utilitária que o produto possui, buscando motivos especiais e úteis para o consumo. Arruda Filho, Cabusas e Dholokia (2008), destacam que produtos com características multifuncionais, possuem a maioria de suas integrações interessantes aos usuários quando estas são predominantemente hedônicas, porém possuem a possibilidade de justificação de uso a partir dos elementos utilitárias intrínsecos que auxiliam na redução do senso de culpa, que os usuários geralmente apresentam quando efetuam a compra de um produto que possuí custo elevado para fins hedônicos.

Logo, os comentários dos participantes expressam percepções hedônicas ligadas ao uso baseado nas tendências que a moda dita, no qual fazem uso do produto como acessórios de beleza proporcionando uma sensação de prazer e de diferenciação social (ARRUDA FILHO; DHOLAKIA, 2013), ao serem vistos utilizando o equipamento.

\subsection{A UTILIDADE PERCEBIDA PELOS CONSUMIDORES DO APPLE WATCH.}

O fator utilitário pode ser observado pelo objetivo de aplicação e uso do Apple Watch, estando relacionado ao uso para o trabalho, estudo ou necessidades laborais dos indivíduos, tendo como objetivo principal o aumento da performance da atividade desenvolvida de forma racional (ARNOLD; REYNOLDS, 2003; BABIN et al., 1994; BATRA; AHTOLA, 1990; DHAR; WERTENBROCH, 2000; HOLBROOK; HIRSCHMAN, 1982), conforme observado no comentário dos indivíduos pesquisados.

Durante o trajeto do trabalho, não iria precisar atender o celular, pois um simples toque de comando posso conversar sem perder a atenção ao dirigir. (Pergunta 2; respondente 38).

Nossas atividades requerem expertises diversas e dia-dia, sendo assim no supermercado, por exemplo, enquanto faço compras posso verificar meus batimentos cardíacos. (Pergunta 2; respondente 50)

[...] os contatos estariam de certa forma conectados ao pulso... o terror de esquecer o celular nos momentos mais impróprios diminuiria bastante [...]. (Pergunta 3; respondente 10)

Conforme Chitturi et al. (2007), a junção de funcionalidade e prazer gera uma grande expressão na utilização de novos produtos tecnológicos, pois seu uso pode gerar uma REAd | Porto Alegre - Vol. 24 - No 3 - Setembro / Dezembro 2018 - p. 244-268 
percepção de bem estar, fazendo com que seu consumo passe a ser justificado pelas funcionalidades que o produto oferece.

A instrumentalidade com o uso e a forma racional, contextualizada nas necessidades pessoais em função do trabalho, interesses pessoais funcionais e garantia da utilidade com o produto, focando em aumento da performance, facilidade de execução de tarefas, eficiência laboral e uso como ferramenta que auxilia na execução de problemas ou acesso a informações, é a descrição que demonstra utilitarismo ao contexto. Logo, percebe-se nos comentários dos consumidores, que os mesmos buscam facilitar suas atividades ou diminuir o esforço relacionado à realização de ações, como por exemplo, atender o celular com apenas um toque no dispositivo ou checar a atividade cardíaca de maneira rápida e eficaz.

\subsection{STATUS SOCIAL COMO FATOR INFLUENCIADOR NO CONSUMO}

Esta categoria de consumidores se mostra motivada a adquirir o Apple Watch devido à forte atmosfera de diferenciação social, que envolve a utilização deste produto. Por isso, os consumidores se sentem como indivíduos diferenciados que podem executar tarefas, utilizar um produto com design moderno, atraente, tecnológico e bonito, além de custar um preço que não é acessível a todas as classes sociais, o que por meio destes fatores concede destaque social aos seus possuidores (DIAS, 2012, BELK, 1988), como pode ser observado nos comentários abaixo.

Eu penso que me enquadraria em outro patamar social pela exclusividade que o produto representa. (Pergunta 7; respondente 7) Reconhecimento por usar um produto apple e altamente inovador com muitos beneficios. (Pergunta 4; respondente 36)

Relógio quase todos têm, entretanto, um relógio que faz ligações, tem GPS e outras funções são poucas pessoas que possuem, inclusive pelo preço (uma parcela de exclusividade. (Pergunta 7; respondente 11)

Seria um dos únicos a ter um relógio como este, com qualidade e com várias funções. (Pergunta 7; respondente 23)

Identifica-se nos participantes analisados que existe a presença de consumidores que se consideram tecnológicos, que aderem a novas tecnologias e gostam de desfrutá-las e estar sempre adequados aos avanços dos dispositivos disponíveis no mercado. Entretanto, existem muitos comentários que demonstram os consumidores buscando ou vislumbrando a 
tecnologia, para destacarem-se ou se incluir em um grupo social distinto. Fortunati (2002) salienta que as dimensões estéticas passam a gerar uma implicação social com possibilidade de impacto sobre o uso de uma determinada tecnologia, ou seja, passa atribuir seu sucesso à "Beleza da Moda". Enquanto Dias (2012), destaca que a autoimagem de um produto pode causar uma influência sobre as pessoas ao verem outras utilizando ou ainda portando um produto tecnológico com aspectos estéticos envolvidos. Diante disto, se observa que os consumidores passam a ter uma percepção de diferenciação, por estarem utilizando um produto como o Apple Watch e de enquadramento em um grupo de pessoas exclusivas e seletas que podem comprar e possuir este tipo de produto.

\subsection{RISCO SOCIAL COMO ELEMENTO QUE INDUZ A NÃO ADOÇÃO DO APPLE WATCH}

O comportamento social exerce uma grande influência sobre o comportamento individual dos consumidores, pois com o lançamento de novas tecnologias $\mathrm{e} o$ aperfeiçoamento de equipamentos já existentes, é necessário que as pessoas passem a se adaptar a este novo contexto tecnológico, buscando informações sobre a usabilidade destes dispositivos (STEG; VELK, 2009).

Neste contexto, são percebidos alguns fatores que geram uma barreira de adoção e/ou aceitação no uso de novas tecnologias, a qual se destaca o risco social, sendo categorizado por Gifford (2011), como uma barreira que pode diminuir ou impedir um comportamento de adoção, quando a influência negativa de outra pessoa gere uma variação no comportamento humano (GASPAR DE CARVALHO, 2004), conforme observado nos comentários a seguir.

Poderia causar um ar de inveja. (Pergunta 5; respondente 8)

Acho que as reações seriam diversas. Penso que umas pessoas ficariam curiosas em conhecer o produto e de repente até pensariam em adquirir um. No entanto, eu imagino que outras pessoas poderiam sentir inveja devido à impossibilidade de adquirir o aparelho pelo preço alto. (Pergunta 6; respondente 7)

Sentiriam inveja. (Pergunta 6; respondente 9)

Achar que tenho boas condições de vida e acabarem se aproveitando disso. (Pergunta 6; respondente 8)

Provocaria inveja e até espanto em alguns. (Pergunta 9; respondente 1)

REAd | Porto Alegre - Vol. 24 - No 3 - Setembro / Dezembro 2018 - p. 244-268 
Amigos e familiares pensariam que tenho muito dinheiro para gastar com equipamentos caros e sofisticados. (Pergunta 7; respondente 29) Cobiçariam o produto por ser algo inovador. (Pergunta 6; respondente 25)

O comportamento do consumidor passa por alterações devido ao risco social que envolve a posse do Apple Watch e a percepção dos indivíduos sobre as motivações que levam o usuário a utilizar este produto. Os comentários indicam que a inveja e a cobiça podem atuar como barreiras, percebidas como prejudiciais ao interesse de possessão e uso do produto. Este cenário pode alterar as práticas de uso e consumo, visto que houveram consumidores que se sentiram ameaçados em aderir ao produto, devidos ao impacto que o mesmo poderia causar a sua imagem na sociedade.

Segundo Cukier (2011) quando nos comparamos com outras pessoas e percebemos que nosso valor, autoestima e autorrespeito ficam diminuídos, sentimos uma dor psicológica que chamamos de inveja. Sendo assim, alguns entrevistados percebem que ao fazer uso de produtos altamente tecnológicos com características diferenciadas das demais, isto acaba por elevar o posicionamento do indivíduo, transformando-o em um alvo para críticas. Devido a isso, surgem conflitos entre pessoas, o que influencia de forma intensa a não adoção do equipamento, em virtude das reações das pessoas e do impacto social que o uso deste produto pode vir a causar na sociedade, na qual o usuário se insere.

\subsection{A PERCEPÇÃO MULTIFUNCIONAL DA CONVERGÊNCIA TECNOLÓGICA}

De acordo com Mukherjree e Hoyer (2001), produtos inovadores como o Apple Watch, atraem grandes expectativas para os consumidores. Os usuários conseguem enxergar vantagens relativas diante ao uso do aparelho, predizendo que o dispositivo auxiliará no desenvolvimento de suas atividades diárias. Logo, as tecnologias convergentes oferecem uma gama de possibilidades de uso, oportunizando aos seus usuários uma maior acessibilidade de recursos, mobilidade, eficiência e redução de tempo na execução de tarefas (GILL, 2008; HARRIS; BLAIR, 2006; KIM; WONJOON, 2018; LEE; PARK; KANG, 2018; NUNES et al., 2000), conforme observado nos comentários dos consumidores.

Comunicação com outra pessoa sem necessitar diretamente de um celular: - localizar-me através do GPS; - Momentos felizes se eu conseguir algum exito com exercícios e o mesmo ser representado pelo cel. (Pergunta 1; respondente 12) 
Dizem que tempo é dinheiro, e otimizá-lo é primordial para o desenvolvimento de nossas atividades, assim as funcionalidades do produto proporcionarão uma otimização deste tempo, onde durante uma atividade física, podemos ter acesso a e-mails e outras funcionalidades sem muito esforço (Pergunta 03; respondente 50).

Pode ajudar a ter um melhor controle da agenda profissional, principalmente no caso do celular descarregar. Além de funcionar como sentinela para manter a saúde em dia. (Pergunta 1; respondente 13)

O prazer de se usar como acessório e desfrutar de multifuncionalidades compactas que ele oferece. (Pergunta 1; respondente 26)

Seria útil em campo para afeição de pontos geográficos para posterior mapeamento da área a ser georreferenciada, entre outras diversas funções, como olhar a hora, falar ao telefone e marcar pontos no GPS ao mesmo tempo com um único aparelho. (Pergunta 2; respondente 13)

Neste ambiente multifuncional a diversidade é um ponto fundamental para aumento dos benefícios percebidos pelos consumidores (LEE; LEE; GARRETT, 2013). Percebe-se que estes usuários acreditam que tal produto lhes trará maior satisfação e resultado, ao usar um equipamento que seja altamente tecnológico e traga consigo múltiplas interfaces que aumentam o valor do dispositivo. Consumidores tecnológicos estão sempre em busca de produtos que facilitem suas atividades e destacam o mesmo por serem produtos diferentes dos que já utilizam (ARRUDA FILHO; CABUSAS; DHOLAKIA, 2010; GILL, 2008).

\section{DISCUSSÃO DOS RESULTADOS}

Objetivando demonstrar a estrutura relacionada a percepção dos consumidores em relação a adoção do Apple Watch, a Figura 1 foi estabelecida, expondo os valores e as relações que impactam no comportamento de consumo para adotar ou não o produto. Assim, conforme apresentado na Figura 1, percebe-se que elementos como fatores utilitários, hedônicos e sociais foram inseridos e coexistem no dispositivo devido à convergência 
tecnológica que permitiu diversas opções integradas. A convergência amplia a percepção de inovação dos usuários, pois estes entendem que por haverem diversas possibilidades de uso, utilizam mais o equipamento, sendo este um fator que estimula a adoção do produto.

Tomando como base o trabalho de Nunes e Arruda Filho (2018) que objetiva identificar os elementos que exercem maior influência sobre a intenção de consumo do dispositivo vestível Google Glass, realizou-se uma adaptação da figura que representa o referido estudo, a fim de expressar de forma clara, os fatores que impactam na adoção do relógio inteligente Apple Watch.

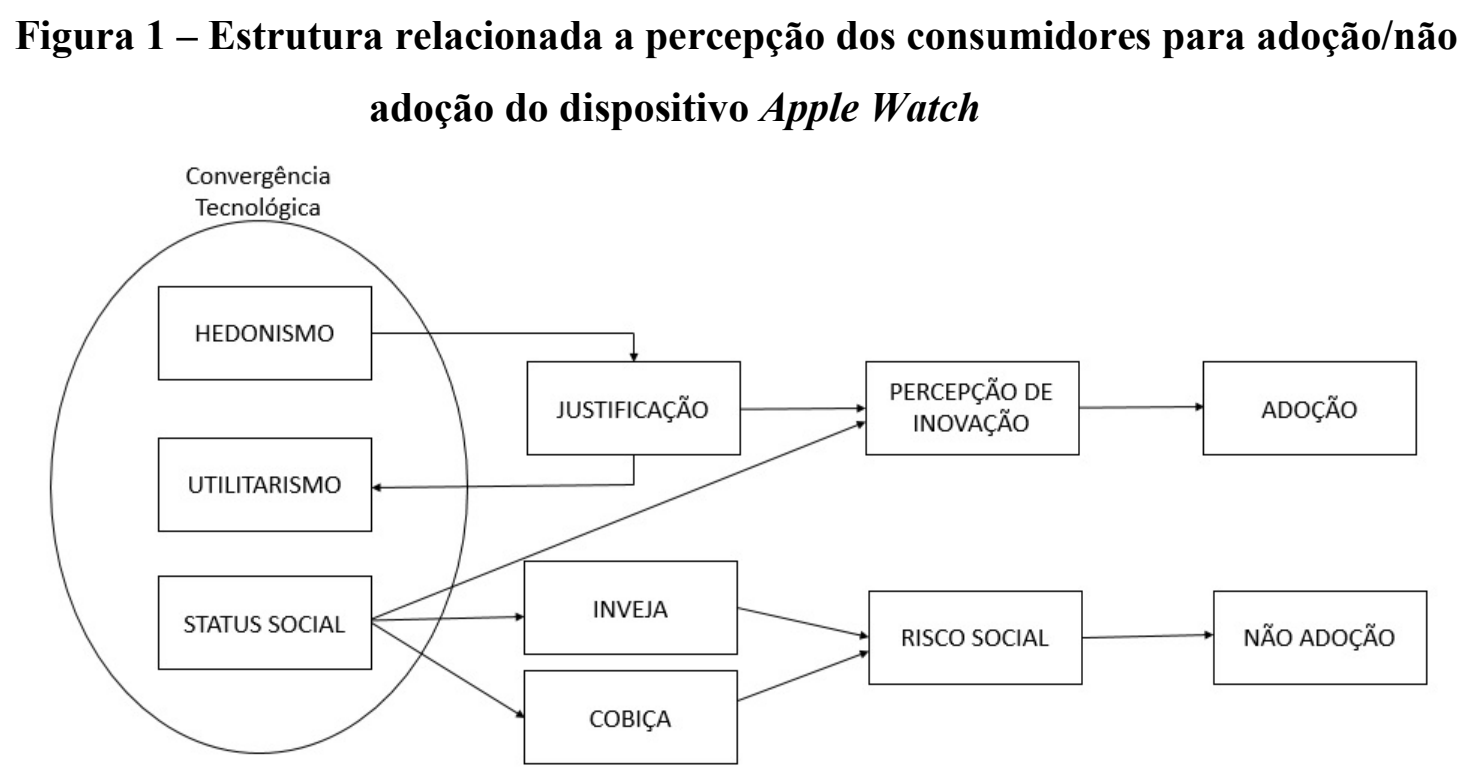

Fonte: adaptado de Nunes e Arruda Filho (2018).

Neste cenário, também se percebe que alguns usuários consideram o Apple Watch por causa de suas características hedônicas. Porém, como este produto possuí um valor monetário e social elevado, estes usuários sentem a necessidade de justificar a adoção por intermédio dos elementos utilitários presentes no dispositivo, a fim de reduzir a culpa que é gerada a partir da adoção de um produto de alto valor agregado para fins hedônicos.

Contudo, muito embora os a utilização do Apple Watch gere status social aos seus possuidores, alguns destes percebem que devido à amplificação dos fatores sociais, determinadas pessoas presentes no convívio social destes usuários se comportam de maneira escusa, expressando comportamentos que retraem o usuário do dispositivo. Logo, fatores como a inveja e a cobiça representam um elevado risco social para os usuários do Apple 
Watch, fazendo com que os indivíduos utilizem pouco ou percebam o dispositivo como um produto inviável a adoção.

\section{CONCLUSÃO}

Este artigo buscou compreender as características comportamentais envolvidas no consumo de produtos tecnológicos vestíveis. $\mathrm{O}$ estudo identificou os fatores que influenciaram na adoção deste tipo específico de tecnologia, tendo como objeto da pesquisa o dispositivo Apple Watch da marca Apple (relógio inteligente), no qual o dispositivo se apresentou como um produto inovador e percebido como altamente tecnológico pelos consumidores, dado o pouco conhecimento e experiência apresentada pelos participantes entrevistados.

A pesquisa foi realizada a partir da aplicação de questionários contendo perguntas abertas, com o objetivo de coletar dados que expressassem os significados relacionados a percepção de uso e possível consumo do Apple Watch. As informações coletadas foram interpretadas por intermédio da análise de conteúdo, almejando categorizar os fatores que poderiam descrever melhor as características dos consumidores de tecnologias vestíveis.

Os resultados obtidos apontam a existência de uma ligação entre os fatores comportamentais identificados e sua influência quanto ao uso e consumo do relógio inteligente Apple Watch, sendo classificado como um produto altamente tecnológico e com multifuncionalidades que agregam diversos valores em função da percepção dos usuários. Dentre os fatores que influenciam na adoção de produtos tecnológicos do tipo vestíveis, destacaram-se a preferência dos usuários pela diferenciação social a partir da obtenção de status, a utilização do dispositivo para fins hedônicos, que concedem momentos de prazer e diversão, podendo a compra ser justificada por motivações utilitárias, sendo estas possibilitadas por intermédio da convergência de diversos elementos funcionais presentes neste dispositivo.

Além disso, a análise dos dados também identificou a inveja e a cobiça como riscos sociais que influenciam na não adoção do produto pelos consumidores, uma vez que os mesmos acreditam ser socialmente arriscado consumir produtos com conceitos altamente tecnológicos, voltados ao luxo, que sejam exclusivos e que possuam preços elevados, devido ao impacto que a posse do dispositivo pode causar a sua imagem na sociedade.

O estudo realizado possuiu limitações como a apresentação de um número reduzido de respondentes. Ocorreram limitações devidas a pesquisa ser de base exploratória com foco REAd | Porto Alegre - Vol. 24 - No 3 - Setembro / Dezembro 2018 - p. 244-268 
em observar o comportamento de consumo, uma vez que este estudo poderia ter utilizado outros instrumentos de coleta e análise de dados para melhor descrever o comportamento de consumo em relação à tecnologia pesquisada. Sendo assim, como sugestão para pesquisas futuras, indica-se realizar a aplicação de métodos diferentes que possam aprofundar o estudo e compreender melhor a barreira de consumo, que foi identificado no trabalho e denominada como Risco Social.

\section{REFERÊNCIAS}

AMADO, J. Manual de investigação qualitativa em educação. Coimbra: Universidade de Coimbra, 2013.

ARAÚJO, J. B. Uma análise do cenário publicitário online no ano em que o mobile ultrapassou o desktop. Intercom. Anais... 2015.

ARNOLD, M. J.; REYNOLDS, K. E. Hedonic shopping motivations. Journal of Retailing, v. 79, n. 2, p. 77-95, 2003.

ARRUDA FILHO, E. J. M. Incluindo o fator social no modelo de aceitação tecnológica para estruturas convergentes. Revista de Administração, v. 43, n. 4, p. 315-330, 2008.

ARRUDA FILHO, E. J. M.; CABUSAS, J. A.; DHOLAKIA, N. 2010. Social behavior and brand devotion among iPhone innovators. International Journal of Information Management, v. 30, n. 6, p. 475-480, 2010.

ARRUDA FILHO, E. J. M.; CABUSAS, J. J.; DHOLAKIA, N. 2008. Fator Social versus Tecnologia Utilitária: Marketing Social versus Mercado Utilitário. Revista de Gestão da Tecnologia e Sistemas de Informação, v. 5, n. 2, p. 305-324, 2008.

ARRUDA FILHO, E. J. M.; DHOLAKIA, R. R. Hedonismo como um Fator de Decisão e Uso Tecnológico. Revista Brasileira de Gestão de Negócios, v. 15, n. 48, p. 343-361, 2013. ARRUDA, D. M. O.; MIRANDA, C. M. C. Variáveis comportamentais determinantes de compra no varejo virtual: um estudo com consumidores brasileiros. Organizações Rurais \& Agroindustriais, v. 5, n. 2, 2003.

BABIN, B. J.; DARDEN, W. R.; GRIFFIN, M. Work and/or fun: measuring hedonic and utilitarian shopping value. Journal of Consumer Research, v. 20, n. 4, p. 644-656. 1994. BARDIN, L. Análise de conteúdo. Lisboa: Edições 70, 1977.

BATRA, R.; AHTOLA, O. T. Measuring the hedonic and utilitarian sources of consumer attitudes. Marketing Letters, v. 2, n. 2, p. 159-170. 1990. 
BELK, Russell W. Extended self in a digital world. Journal of Consumer Research, v. 40, n. 3, p. 477-500, 2013.

BELK, R, W. Possessions and the extended self. Journal of consumer research, v. 15, n. 2, p. 139-168, 1988.

BOGDAN, R.; BIKLEN, S. Investigação qualitativa em educação: uma introdução à teoria e os métodos. Porto: Porto Editora. 1994.

CHITTURI, R.; RAGHUNATHAN, R.; MAHAJAN, V. Form Versus Function: How the Intensities of Specific Emotions Evoked in Functional Versus Hedonic Trade-Offs Mediate Product Preferences. Journal of Marketing Research, v. 44, p. 702-714, 2007.

CUKIER, R. Psicossociodrama da inveja: atire a primeira pedra se você puder! Revista Brasileira de Psicodrama, v. 19, n. 1, p. 13-33. 2011.

DEHGHANI, Milad; KIM, Ki Joon; DANGELICO, Rosa Maria. Will smartwatches last? Factors contributing to intention to keep using smart wearable technology. Telematics and Informatics, v. 35, n. 2, p. 480-490, 2018.

DHAR, R.; WERTENBROCH, K. Consumer choice between hedonic and utilitarian goods. Journal of Marketing Research, v. 37, n. 1, p. 60-71. 2000.

DHAR, Sujoy. Humanity and Technology: The Alliance. 2018.

DIAS, Edgar José Pereira. Comportamento do consumidor no setor educacional: adoção e aceitação de novas tecnologias. 124 f. Dissertação (Mestrado em Administração) Universidade da Amazônia, Belém - PA. 2012.

DONATI, L. P. Computadores vestíveis: convivência de diferentes especialidades. Conexão - Comunicação e Cultura, v. 3, n. 6, p. 93-102, 2010.

EHRENBERG, K. C. O. Consumo simbólico e a vida cotidiana. II ECOM, Anais... 2012. FARIAS FILHO, M. C.; ARRUDA FILHO, E. J. M. Planejamento da pesquisa científica. São Paulo: Atlas, 2013.

FARIAS, C.; DUSCHITZ, C.; CARVALHO, G.M. Marketing aplicado. Porto Alegre: Bookman, 2014.

FORTUNATI, L. Perpetual contact: mobile communication, private talk, public performance. Cambridge: Cambridge University Press, 2002.

FRANCO, P. B. Um estudo para o desenvolvimento de peças de vestuário com sensores têxteis incorporados com a função de prevenir má postura corporal. Dissetação de Mestrado apresentada na Faculdade de Engenharia da Universidade da Beira Interior, 2013. 
GASPAR DE CARVALHO, R. Concepção de projectos de Educação Ambiental [Conception of Environmental Education Projects]. Manual do curso de "Animadores de Educação Ambiental. Montijo, Portugal: EPMIEFP. 2004.

GIFFORD, R. The dragons of inaction: Psychological barriers that limit climate change mitigation and adaptation. American Psychologist, n. 66, p. 290-302. 2011.

GILL, T. Convergent products: what functionalities add more value to the base? Journal of Marketing, v. 72, n. 2, p. 46-62, mar. 2008.

GUPTA, V.; RODRIGUES, L. L. R; MATHEW, O. Identifying opportunities for wearable technology for product development and market positioning. International Journal of Product Development, v. 22, n. 4, p. 247-275, 2018.

HANNEMAN, G. J; CARROLL, T. W; ROGERS, E. M; STANFIELD, J. D.; LIN, N. Computer Simulation of Innovation Diffusion In A Peasant Village. The American, v. 12, n. 6, Jul/Aug. 1969.

HARRIS, J.; BLAIR, E. A. Functional compatibility risk and consumer preference for product bundles. Journal of the Academy of Marketing Science, v. 34, n. 1, p. 19-26, 2006. HEIN, D. W.; JODOIN, J. L.; RAUSCHNABEL, P. A.; IVENS, B. S. Are wearables good or bad for society? An exploration of societal benefits, risks, and consequences of augmented reality smart glasses. In: Wearable Technologies: Concepts, Methodologies, Tools, and Applications. Hershey: IGI Global,2018, p. 1313-1337.

HOLBROOK, M. B.; HIRSCHMAN, E. C. The experiential aspects of consumption: consumer fantasies, feelings and fun. Journal of Consumer Research, v. 9, n. 2, p. 132141. 1982.

IBGE. PNAD 2013 (Pesquisa Nacional por Amostra de Domicílios). Acesso à Internet e à Televisão e Posse de Telefone Móvel Celular para Uso Pessoal 2013. Rio de Janeiro: IBGE (Instituto Brasileiro de Geografia e Estatística), 2015. JOEP W. C. ARTS; RUUD T. FRAMBACH; TAMMO H. A. BIJMOLT. Generalizações sobre a adoção da inovação do consumidor: Uma meta análise sobre os condutores de intenção e comportamento. Intern. J. of Research in Marketing, v. 28, p. 134-144. 2011. KATZ, J. E. SUGIYAMA, S. Mobile phones as fashion statements: evidence from student surveys in the US and Japan. New Media \& SOCIETY, v. 8, n. 2, p. 321-337, 2006. KIM, K.; KIM, W. Does Convergence Matter? The Effect of Technology Convergence on Firms Market Performance. Academy of Management Global Proceedings, (published first online) 2018. 
LEE, Chul; PARK, Gunno; KANG, Jina. The impact of convergence between science and technology on innovation. The Journal of Technology Transfer, v. 43, n. 2, p. 522-544, 2018 .

LEE, Misuk; CHO, Youngsang. Consumer perception of a new convergence product: A theoretical and empirical approach. Technological Forecasting and Social Change, v. 92, p. 312-321. 2015.

LEE, S.; LEE, J.-H.; GARRETT, T. C. A study of the attitude toward convergent products: a focus on the consumer perception of functionalities. Journal of Product Innovation Management, v. 30, n. 1, p. 123-135, 2013.

LORENZETTI, Jorge et al. Tecnologia, inovação tecnológica e saúde: uma reflexão necessária. Texto and Contexto Enfermagem, v. 21, n. 2, p. 432, 2012.

MICK, David Glen; FOURNIER, Susan. Paradoxes of technology: Consumer cognizance, emotions, and coping strategies. Journal of Consumer research, v. 25, n. 2, p. 123-143, 1998.

MIRANDA, C. M. C.; ARRUDA, D. M. O. Produtos e variáveis comportamentais determinantes de compra no varejo virtual: um estudo com consumidores brasileiros. Revista Eletrônica de Administração, v. 10, n. 1, p. 1-16, 2004.

MUKHERJEE, A.; HOYER, W. D. The Effect of Novel Attributes on Product Evaluation. Journal of Consumer Research, v. 28, December. 2001.

NASCIMENTO, Bruno; OLIVEIRA, Tiago; TAM, Carlos. Wearable technology: What explains continuance intention in smartwatches? Journal of Retailing and Consumer Services, v. 43, p. 157-169, 2018.

NIERODA, M. E.; MRAD, M.; SOLOMON, M. R. How do consumers think about hybrid products? Computer wearables have an identity problem. Journal of Business

Research, v.89, p. 159-170, 2018.

NUNES, P.; WILSON, D.; KAMBIL, A. The all-in-one market. Harvard Business Review, v. 78, n. 3, p. 19-20. 2000.

NUNES, G. S.; ARRUDA FILHO, E. J. M. Consumer behavior regarding wearable technologies: Google Glass. Innovation \& Management Review, v. 15, n. 3, p. 230-246, 2018.

OKADA, Erica Mina. Justification effects on consumer choice of hedonic and utilitarian goods. Journal of marketing research, v. 42, n. 1, p. 43-53. 2005. 
OZCAN, T.; SHEININ, D. A. The effects of changing attribute composition on judgments about multifunctional products. Journal of Marketing Theory and Practice, v. 23, n. 1, p. 107-118, 2015.

PAGE, Tom. A Forecast of the Adoption of Wearable Technology.International Journal of Technology Diffusion (IJTD), v. 6, n. 2, p. 12-29. 2015.

PANISSON, L. S.Dos Computadores Vestíveis às Roupas Pensantes: Os Avanços

Tecnológicos a Serviço da Moda e da Comunicação. Simpósio Nacional ABCIBER, v. 3. Anais... 2009.

ROGERS, M. Difusion of innovation. New York: Free Press, 2003.

SCHERER, F. Como as tecnologias vestíveis estão criando novas oportunidades.

Disponível em: http:/exame.abril.com.br/rede-de-blogs/inovacao-na-pratica/2014/11/05/ como-as-tecnologias-vestiveis-estao-criando-novas-oportunidades/. Acesso em: 20 mai. 2015 .

STEG, L.; VLEK, C. Encouraging pro-environmental behaviour: An integrative review and research agenda. Journal of environmental psychology, v. 29, n. 3, p. 309-317. 2009.

TENUTA, L.; TESTA, S. Scientific method and creative process for wearable technologies from invention to innovation. Airea: Arts and Interdisciplinary Research, n. 1, p. 35-46, 2018.

VOSS, K. E.; SPANGENBERG E. R.; GROHMANN, B. Measuring the Hedonic and Utilitarian Dimensions of Consumer Attitude. Journal of Marketing Research, v. 40, p. 310-40, August. 2003.

WOOD, J. Revolutions in wearable technology for apparel. In: MCLOUGHLIN, J.; SABIR, T. (Eds.) High-Performance Apparel. London: Elsiever, 2017, p. 325-339.

WOOD, S. A. Framing Wearing: Genre, Embodiment, and Exploring Wearable Technology in the Composition Classroom. Computers and Composition. 2018.

YOFFIE, D.B. Competing in the age of digital convergence. Harvard Business Press. 1997. 